\title{
Intra-breath measures of respiratory mechanics in healthy African infants detect risk of respiratory illness in early life
}

\begin{abstract}
Diane M. Gray ${ }^{1,10}$, Dorottya Czovek ${ }^{2,3,10}$, Lauren McMillan ${ }^{1}$, Lidija Turkovic ${ }^{4}$, Jacob A.M. Stadler (10 ${ }^{1}$, Anessa Vanker ${ }^{1}$, Bence L. Radics ${ }^{5}$, Zoltán Gingl ${ }^{6}$, Graham L. Hall ${ }^{4,7,8}$, Peter D. Sly ${ }^{2}$, Heather J. Zar ${ }^{1,11}$ and Zoltán Hantos $5,9,11$

Affiliations: ${ }^{1}$ Dept of Paediatrics and Child Health, Red Cross War Memorial Children's Hospital and MRC Unit on Child and Adolescent Health, University of Cape Town, Cape Town, South Africa. ${ }^{2}$ Child Health Research Centre, University of Queensland, Brisbane, Australia. ${ }^{3} 1 \mathrm{st}$ Dept of Paediatrics, Semmelweis University, Budapest, Hungary. ${ }^{4}$ Telethon Kids Institute, Perth, Australia. ${ }^{5}$ Dept of Pulmonology, University of Szeged, Szeged, Hungary. ${ }^{6}$ Dept of Technical Informatics, University of Szeged, Szeged, Hungary. ${ }^{7}$ School of Physiotherapy and Exercise Science, Curtin University, Perth, Australia. ${ }^{8}$ Centre for Child Health, University of Western Australia, Perth, Australia. ${ }^{9}$ Dept of Anaesthesiology and Intensive Therapy, Semmelweis University, Budapest, Hungary. ${ }^{10}$ These two authors are joint first authors. ${ }^{11}$ These two authors are joint senior authors.
\end{abstract}

Correspondence: Zoltán Hantos, Dept of Anaesthesiology and Intensive Therapy, Semmelweis University, 78/B Üllői út, 1082 Budapest, Hungary. E-mail: hantos.zoltanamed.u-szeged.hu

@ERSpublications

Novel measurements of respiratory mechanics are feasible in infants in a community setting and able to detect changes in lung function in healthy infants associated with increased risk of subsequent LRTI in infancy http://ow.ly/IUKk30mCfi3

Cite this article as: Gray DM, Czovek D, McMillan L, et al. Intra-breath measures of respiratory mechanics in healthy African infants detect risk of respiratory illness in early life. Eur Respir J 2019; 53: 1800998 [https://doi.org/10.1183/13993003.00998-2018]

ABSTRACT Lower respiratory tract illness (LRTI) is a leading cause of mortality and morbidity in children. Sensitive and noninvasive infant lung function techniques are needed to measure risk for and impact of LRTI on lung health. The objective of this study was to investigate whether lung function derived from the intra-breath forced oscillation technique (FOT) was able to identify healthy infants at risk of LRTI in the first year of life.

Lung function was measured with the novel intra-breath FOT, in 6-week-old infants in a South African birth cohort (Drakenstein Child Health Study). LRTI during the first year was confirmed by study staff. The association between baseline lung function and LRTI was assessed with logistic regression and odds ratios determined using optimal cut-off values.

Of the 627 healthy infants with successful lung function testing, 161 (24\%) had 238 LRTI episodes subsequently during the first year. Volume dependence of respiratory resistance $(\Delta R)$ and reactance $(\Delta X)$ was associated with LRTI. The predictive value was stronger if LRTI was recurrent $(\mathrm{n}=50$ (31\%): OR 2.5, $\Delta X)$, required hospitalisation $(\mathrm{n}=38(16 \%)$ : OR 5.4, $\Delta R)$ or was associated with wheeze $(\mathrm{n}=87(37 \%)$ : OR 3.9, $\Delta X)$.

Intra-breath FOT can identify healthy infants at risk of developing LRTI, wheezing or severe illness in the first year of life.

This article has supplementary material available from erj.ersjournals.com

Received: May 292018 | Accepted after revision: Nov 012018

Copyright $\odot$ ERS 2019. This article is open access and distributed under the terms of the Creative Commons Attribution Licence 4.0. 


\section{Introduction}

Low lung function and lower respiratory tract illness (LRTI) in early life are both associated with increased risk of respiratory disease in later life [1-3]. LRTI remains a leading cause of morbidity and mortality in childhood globally [4-6], particularly in low- and middle-income countries [5, 6], potentially contributing to the burden of later chronic respiratory disease [7]. The association of reduced lung function soon after birth and subsequent risk of wheezing in childhood has been shown in a number of cohort studies $[1,2,8]$. Whether baseline lung function increases risk for severe LRTI and LRTI not associated with wheeze is not as clear [9]. Using standard lung function techniques we have previously published that LRTIs in infancy reduce lung function in early childhood in an African birth cohort, an effect independent of baseline lung function [10], suggesting that both baseline lung function and LRTI are important in determining later respiratory lung function and health. Being able to accurately measure lung function in unsedated infants is possible [11]. Ideally, the measure should be sensitive enough to pick up small changes in lung function and be able to comprehensively characterise the mechanics of the respiratory system. The forced oscillation technique (FOT) has been successfully used in unsedated infants and is able to detect the impact of antenatal exposures on early-life lung function [12]. The standard FOT calculates the average respiratory system resistance $(R)$ or reactance $(X)$ during a period of quiet tidal breathing. This method is not able to detect the changes in $R$ and $X$ that occur within the breathing cycle and which may be sensitive measures of respiratory function alterations. This intra-breath analysis of respiratory mechanics is a novel method that has been shown to detect airway obstruction [13] and mechanical inhomogeneity $[13,14]$ in both children and adults, but it has not been used in infants.

Therefore, we aimed to investigate whether lung function derived from the intra-breath FOT performed shortly after birth was able to identify healthy infants at risk of LRTI in the first year of life.

\section{Methods}

The Drakenstein Child Health Study is a prospective birth cohort conducted in a low socioeconomic population in South Africa, with a high incidence of LRTI [15]. Mothers were enrolled antenatally and infants were followed up at scheduled study visits. Strong surveillance systems for LRTI were used [16, 17] and all episodes of LRTI were assessed by trained study staff. Wheezing was diagnosed on auscultation. LRTI was defined by the World Health Organization clinical case definitions: an episode of LRTI (cough or difficulty breathing and increased respiratory rate or lower chest wall in-drawing in a child aged $>2$ months) or severe LRTI (child aged $<2$ months with increased respiratory rate or lower chest wall in-drawing, or any general danger sign in a child of any age) [18].

Antenatal maternal smoking status was determined based on urine cotinine levels [19]. Maternal stress and alcohol intake during pregnancy were assessed at 28-32 weeks of gestation [20].

The study was approved by the Faculty of Health Sciences, Human Research Ethics Committee, University of Cape Town (401/2009) and the Western Cape Provincial Health Research Committee (Western Cape, South Africa). Mothers gave informed, written consent in their first language for participation.

\section{Lung function testing}

Lung function was performed at the 6-week study visit in unsedated infants during quiet sleep. Respiratory impedance $(Z \mathrm{rs})$ was measured with a custom-made wave-tube FOT device [21]. Two different FOT tests were performed. First, the conventional measurement of $Z$ rs spectra using a pseudo-random signal between 8 and $48 \mathrm{~Hz}$ was used. From at least three reproducible 30-s Zrs recordings, mean values of respiratory resistance $(R r s)$ and compliance $(C r s)$ were obtained with model fitting $[12,21]$. Second, a single $16-\mathrm{Hz}$ tracking signal was used in a 60 -s measurement to follow the intra-breath changes in $Z$ rs. Respiratory resistance $(R)$ and reactance $(X)$ at $16 \mathrm{~Hz}$ were determined as functions of time, flow $\left(V^{\prime}\right)$ and volume $(V)$, as published previously $[13,14]$. The intra-breath indices derived from the steady-state segments of the $16-\mathrm{Hz}$ recordings illustrated in figure 1 are summarised and defined in table 1.

\section{Statistical analysis}

Multivariate logistic regression was used to model the predictive ability of lung function variables measured at 6 weeks on the subsequent development of LRTI episode(s) within the first year of life. Antenatal confounding variables defined earlier for the same population were assessed [11]. The determinants included in the final model were: sex, length-for-age $z$-score, ethnicity, maternal smoking during pregnancy, maternal HIV status and gestational age [11]. Pairwise interactions were also explored. Determinants of the intra-breath FOT variables were established (supplementary table S1).

Data from children who had LRTI episode(s) prior to the lung function test or who were lost to follow-up were excluded from the analysis. Logistic regression was performed using four outcomes separately: 1) any LRTI episode, 2) recurrent LRTI, 3) hospitalised LRTI episode and 4) LRTI with wheeze during the first 
year of life. Number of LRTI episodes (recurrent LRTI) was modelled using Poisson regression. Odds ratios, incidence rate ratios, corresponding 95\% confidence intervals and p-values are presented.

Lung function variables shown to be associated with LRTI in the logistic regression were further investigated for predictive ability. Receiver operating characteristic analysis was used to define the optimal
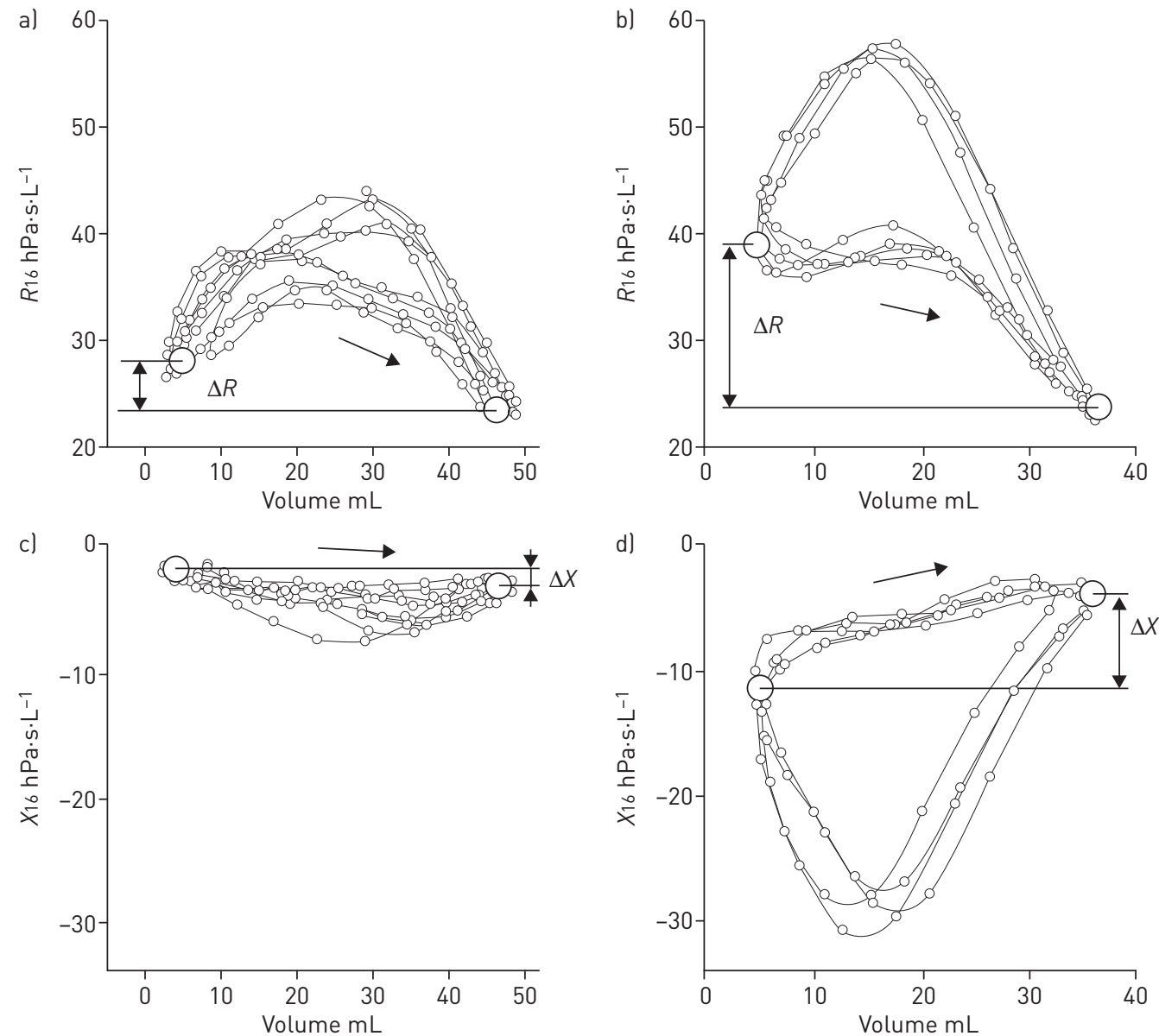

FIGURE 1 Illustration of intra-breath changes derived from the steady-state segments of the $16-\mathrm{Hz}$ recordings of $a, b)$ resistance $(R 16)$ and $c, d)$ reactance $\left(X_{16}\right)$ in $\left.a, c\right)$ an infant who did not develop lower respiratory tract illness (LRTI) and b, d) an infant who did develop LRTI. Arrows indicate the inspiratory limbs. The differences between end-expiratory and end-inspiratory values of $R(\Delta R)$ and $X(\Delta X)$ are also shown. Note the enhanced $\Delta R$ and the opposite change in $\Delta X$ in the infant who did develop LRTI (b and d); also note the large area within the reactance versus volume loops (AXV) indicating expiratory flow limitation in this subject (d).

TABLE 1 Definitions of intra-breath impedance variables derived from the $16-\mathrm{Hz}$ measurements

\begin{tabular}{|c|c|c|}
\hline Variable & Definition & Units \\
\hline $\operatorname{Re}$ & Respiratory resistance calculated for the expiratory phase & $h P a \cdot s \cdot L^{-1}$ \\
\hline$R$ & Respiratory resistance calculated for the inspiratory phase & $h P a \cdot s \cdot L^{-1}$ \\
\hline Rei & Respiratory resistance at the end of inspiration (zero-flow) & $\mathrm{hPa} \cdot \mathrm{S} \cdot \mathrm{L}^{-1}$ \\
\hline Ree & Respiratory resistance at the end of expiration (zero-flow) & $\mathrm{hPa} \cdot \mathrm{s} \cdot \mathrm{L}^{-1}$ \\
\hline$X_{e}$ & Respiratory reactance calculated for the expiratory phase & $h P a \cdot s \cdot L^{-1}$ \\
\hline$X_{i}$ & Respiratory reactance calculated for the inspiratory phase & $h P a \cdot s \cdot L^{-1}$ \\
\hline$X_{\mathrm{ei}}$ & Respiratory reactance at the end of inspiration (zero-flow) & $\mathrm{hPa} \cdot \mathrm{S} \cdot \mathrm{L}^{-1}$ \\
\hline Xee & Respiratory reactance at the end of expiration (zero-flow) & $h P a \cdot s \cdot L^{-1}$ \\
\hline$\Delta R$ & $\begin{array}{c}\text { Volume dependence of respiratory resistance ( } \Delta R=\text { changes in resistance between } \\
\text { the beginning and end of inspiration) }\end{array}$ & $\mathrm{hPa} \cdot \mathrm{s} \cdot \mathrm{L}^{-1}$ \\
\hline$\Delta x$ & $\begin{array}{r}\text { Volume dependence of respiratory reactance ( } \Delta X=\text { changes in reactance between the } \\
\text { beginning and end of inspiration) }\end{array}$ & $h P a \cdot s \cdot L^{-1}$ \\
\hline AXV & Area within the reactance versus volume loops & $\mathrm{hPa} \cdot \mathrm{s}^{-1}$ \\
\hline
\end{tabular}


cut-off values (supplementary table S2). Using the established cut-off values, odds ratios and 95\% confidence intervals were presented.

Data are reported as median (interquartile range). A p-value $<0.05$ was considered as statistically significant. Statistical analysis was performed using Stata version 14 (StataCorp, College Station, TX, USA).

\section{Results}

Of the 912 infants who attended the 6-week visit, FOT testing was successful in 719 (78\%) (figure 2). Data from 54 infants who had any LRTI episode prior to the lung function test and 38 infants who were lost to follow-up before 1 year of follow-up completed were excluded, providing 627 infants (315 male (50.2\%)) with lung function and LRTI information for this analysis. Of these, 161 (24.4\%) children had a total of 238 episodes of LRTI during the first year of life, with an LRTI incidence of 0.45 (95\% CI 0.4-0.5) events per child-year for the first year of life. From the 238 LRTI episodes, 19 (8\%) were severe with a case fatality ratio of $0.4 \%$ (one death), $38(16.0 \%)$ required hospitalisation and $87(36.6 \%)$ were associated with wheeze. 50 (33\%) children had more than one episode of LRTI.

All infants were healthy and symptom-free at the time of lung function testing. There was no difference in gestational age, weight or length at birth between the infants who developed LRTI $(n=161)$ compared with those who did not $(n=466)$; however, males were over-represented in the LRTI group (table 2). There was no significant difference in the prevalence of antenatal exposures that may affect lung function at 6 weeks of age, such as maternal smoking or maternal HIV status (table 2). As a result of the successful prevention of mother-to-child transmission programme there was only one HIV-infected infant in this cohort. Antenatal determinants of the intra-breath FOT measures were similar to previous findings for the standard infant lung function measures in this cohort [11], being associated with sex, infant size and maternal HIV status (supplementary table S1). The volume dependence of $R(\Delta R)$ and $X(\Delta X)$ as well as $X$ e was associated with maternal HIV status but not with maternal smoking or alcohol intake during pregnancy. The unadjusted differences in lung function outcomes between those infants who did and did not experience LRTI in the first year of life are summarised in table 3. In the univariate analysis, $\Delta R, \Delta X$,

FIGURE 2 Description of cohort tested and included in analysis. FOT: forced oscillation technique; LRTI: lower respiratory tract illness.

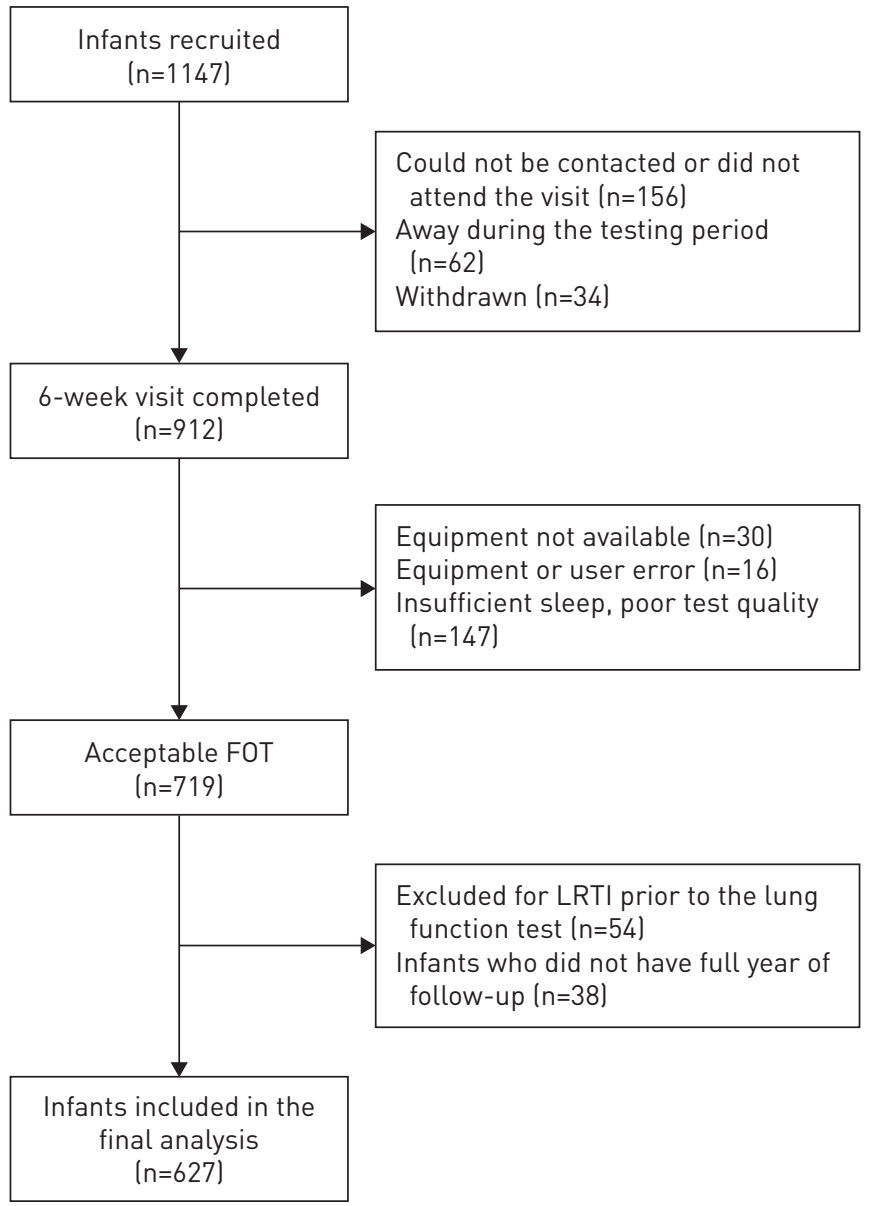


$X \mathrm{e}, \mathrm{Ree}, X_{\mathrm{ee}}$ and area within the reactance versus volume loops (AXV) were significantly different between infants who did and did not develop LRTI.

The reproducibility of the intra-breath impedance measures was assessed by the intra-individual coefficients of variation $(\mathrm{CoV}=\mathrm{SD} /$ mean), where the corresponding mean values of impedance magnitude

TABLE 2 Description of anthropometry and antenatal exposures measured at 6 weeks of age in infants who did not have lower respiratory tract illness (LRTI) and infants who did have LRTI during the first year of life

\begin{tabular}{lccc} 
& No LRTI & LRTI & p-value \\
\hline Subjects & 466 & 161 & \\
Anthropometry & & & 0.633 \\
Gestational age weeks & $39(38-40)$ & $39(37-40)$ & $0.002^{*}$ \\
Male & $217(46.6)$ & $88(60.9)$ & 0.890 \\
Black African ethnicity & $243(52.2)$ & $83(51.6)$ & 0.052 \\
Length-for-age z-score & $-0.73(-1.6-0.11)$ & $-0.88(-1.9--0.04)$ & 0.155 \\
Weight-for-age z-score & $-0.23(-0.94-0.43)$ & $-0.39(-1.15-0.41)$ & 0.944 \\
BMI-for-age z-score & $0.17(-0.56-1.02)$ & $0.21(-0.62-0.97)$ & 0.235 \\
Antenatal exposures & & & 0.089 \\
Maternal antenatal smoking & $336(72.1)$ & $128(79.5)$ & 0.629 \\
$\quad$ Active & $134(30.3)$ & $64(41.6)$ & 0.361 \\
$\quad$ Passive & $202(45.7)$ & $64(41.6)$ & 0.982 \\
$\quad$ No exposure & $106(24.0)$ & $26(16.9)$ & 0.682 \\
$\quad$ No data & $24(5.2)$ & $7(4.3)$ & 0.702 \\
Maternal HIV infection & $96(19.0)$ & $36(22.2)$ & $19(13.5)$ \\
Maternal alcohol intake & $46(10.2)$ & & 238 \\
LRTI episodes & & $50 / 161$ & $19 / 238$ \\
Number of episodes & & $38 / 238$ & $87 / 238$ \\
>1 LRTI episodes (number of infants) & & & \\
Severe LRTI & & & \\
Infants hospitalised for LRTI & & &
\end{tabular}

Data are presented as $n$, median (interquartile range), $n(\%)$ or $n / N$, unless otherwise stated. BMI: body mass index. *: $p<0.05$.

TABLE 3 Lung function measured at 6 weeks of age in infants who did not have lower respiratory tract illness (LRTI) and infants who did have LRTI during the first year of life

\begin{tabular}{|c|c|c|c|}
\hline Lung function & No LRTI & LRTI & p-value ${ }^{\#}$ \\
\hline Subjects & 466 & 161 & \\
\hline Respiratory rate breaths $\cdot \min ^{-1}$ & $46.8(40.2-54.0)$ & $49.1(42.2-57.6)$ & $0.027 *$ \\
\hline Rrs hPa.S.L-1 & $44.3(37.3-54.0)$ & $48.3(37.5-57.0)$ & 0.156 \\
\hline Crs $\mathrm{mL} \cdot \mathrm{hPa}^{-1}$ & $0.99(0.75-1.28)$ & $0.88(0.68-1.21)$ & 0.075 \\
\hline $\operatorname{RehPa} \cdot \mathrm{s} \cdot \mathrm{L}^{-1}$ & $51.9(42.7-63.9)$ & $53.7(43.5-66.8)$ & 0.485 \\
\hline$R \mathrm{hPa} \cdot \mathrm{S} \cdot \mathrm{L}^{-1}$ & $46.8(37.2-60.6)$ & $48.0(38.3-60.3)$ & 0.702 \\
\hline$R e i \mathrm{hPa} \cdot \mathrm{s} \cdot \mathrm{L}^{-1}$ & $37.4(29.9-47.3)$ & $38.3(31.4-50.2)$ & 0.096 \\
\hline Ree hPa.s.L ${ }^{-1}$ & $42.5(36.2-50.8)$ & $43.9(37.5-53.8)$ & $0.009^{*}$ \\
\hline$X \mathrm{hPa} \cdot \mathrm{s} \cdot \mathrm{L}^{-1}$ & $-8.85(-15.04--4.97)$ & $-12.59(-18.98--6.92)$ & $<0.001 *$ \\
\hline$X_{i} \mathrm{hPa} \cdot \mathrm{s} \cdot \mathrm{L}^{-1}$ & $-5.12(-8.89--1.03)$ & $-5.15(-9.97--1.90)$ & 0.405 \\
\hline Xei hPa.s.L $L^{-1}$ & $-4.90(-7.58--2.24)$ & $-5.44(-8.60--2.73)$ & 0.191 \\
\hline Xee hPa.s.L $\mathrm{L}^{-1}$ & $-5.69(-9.83--2.24)$ & $-8.88(-14.06--3.98)$ & $<0.001 *$ \\
\hline$\Delta R \mathrm{hPa} \cdot \mathrm{s} \cdot \mathrm{L}^{-1}$ & $4.43(0.65-8.13)$ & $5.61(1.61-11.21)$ & $0.007^{*}$ \\
\hline$\Delta X \mathrm{hPa} \cdot \mathrm{s} \cdot \mathrm{L}^{-1}$ & $-1.02(-4.69-1.43)$ & $-2.96(-7.52-0.53)$ & $<0.001 *$ \\
\hline AXV hPa.s & $0.18(-0.03-0.49)$ & $0.23(0.03-0.69)$ & $0.011^{*}$ \\
\hline
\end{tabular}

Results presented as $\mathrm{n}$ or median (interquartile range), unless otherwise stated. Rrs: respiratory resistance; Crs: respiratory compliance; Re: expiratory resistance; $R$ i: inspiratory resistance; Rei: respiratory resistance at the end of inspiration; Ree: respiratory resistance at the end of expiration; $X$ e: expiratory reactance; $X_{i}$ : inspiratory reactance; $X_{\mathrm{ei}}$ : respiratory reactance at the end of inspiration; $X_{\mathrm{ee}}$ : respiratory reactance at the end of expiration; $\Delta R$ : volume dependence of resistance; $\Delta X$ : volume dependence of reactance; $A X V$ : area within the reactance versus volume loops. ${ }^{\#}$ : $p$-value of the univariate analysis. ${ }^{*}: p<0.05$. 
were considered to avoid the division by the often near-zero values of $X$. The results of this comparison (supplementary table S3) indicate that the $\mathrm{CoV}$ of the zero-flow readings of $R$ and $X$ are $\sim 2$ times higher and of their respective differences $(\Delta R$ and $\Delta X)$ are 3-4 times higher than the CoV of $R$ mean and $X$ mean.

The results of the logistic regression analysis of lung function variables and LRTI in the first year of life are summarised in table 4 . When values were adjusted for sex, length-for-age, ethnicity, maternal smoking, maternal HIV status and gestational age, $X \mathrm{e}, \Delta R, \Delta X$ and AXV were significantly and independently associated with the development of any LRTI episode (table 4). The associations of $X \mathrm{e}, \Delta R, \Delta X$ and AXV with LRTI were stronger in infants who were hospitalised for LRTI, who had more than one episode of LRTI or who had LRTI with wheeze, and included association with Ree and Xee (table 4).

The cut-off values of the relevant lung function measures and the odds ratios for predicting LRTI in the first year of life are given in table 5. $\Delta X$ and $\Delta R$ exhibited the strongest predictive values for LRTI during the first year of life: infants with $\Delta X$ below $-2.47 \mathrm{hPa} \cdot \mathrm{s} \cdot \mathrm{L}^{-1}$ or $\Delta R$ above $11.06 \mathrm{hPa} \cdot \mathrm{s} \cdot \mathrm{L}^{-1}$ had 2.3 and 2.6 times greater odds of having LRTI, respectively. This effect was independent of the time between the lung function measurement and the first LRTI episode $(\mathrm{r}=0.05 ; \mathrm{p}=0.594)$. The odds ratios were increased if infants required hospitalisation $(\Delta X$ : OR 3.7; $\Delta R$ : OR 5.4) or episodes were recurrent ( $\Delta X: \mathrm{OR} 2.5 ; \Delta R$ : OR 2.4). The intra-breath FOT values $X \mathrm{e}, \Delta X, \Delta R$ and AXV were all predictive of wheezing LRTI (table 5).

\section{Discussion}

The novel intra-breath FOT measures were strongly associated with subsequent clinical expression of LRTI in this South African birth cohort. The association of altered lung function and LRTI risk was even stronger for recurrent LRTI, for severe LRTI as reflected by hospitalisation and for LRTI with wheeze. These associations were independent of gestational age, sex, body length, ethnicity or antenatal exposures.

This is the first study to use the intra-breath analysis of respiratory mechanics in an infant cohort, establishing new outcome measures based on end-expiratory and end-inspiratory impedance data with acceptable reproducibility. In pre-school-age children with acute and recurrent wheeze, an increased volume dependence of $R(\Delta R)$ was identified as a measure of airway obstruction [13], and $\Delta R$ was increased in adults with COPD [14]. In the present study, $\Delta R$ was elevated in infants who later developed LRTI, which suggests the presence of airway obstruction in apparently healthy infants who had a higher risk for LRTI. This is also supported by the fact that measures that have been shown to be related to airflow limitation (e.g. AXV and $X \mathrm{e}$ ) were also abnormal in infants with subsequent LRTI [14, 22]. The change in $X$ during inspiration $(\Delta X)$ has been found to be altered in children with asthma [13] and adults

TABLE 4 Logistic regression analysis of the lung function variables associated with lower respiratory tract illness (LRTI) during the first year of life

\begin{tabular}{|c|c|c|c|c|c|c|c|}
\hline \multicolumn{2}{|c|}{ Any LRTI" } & \multicolumn{2}{|c|}{ Recurrent LRTI } & \multicolumn{2}{|c|}{ Hospitalised LRTI+ } & \multicolumn{2}{|c|}{ Wheezy LRTI§ } \\
\hline $\begin{array}{c}\text { Coefficient } \\
(95 \% \mathrm{CI})\end{array}$ & p-value & $\begin{array}{c}\text { Coefficient } \\
(95 \% \text { CI) }\end{array}$ & p-value & $\begin{array}{c}\text { Coefficient } \\
\text { (95\% CI) }\end{array}$ & p-value & $\begin{array}{c}\text { Coefficient } \\
\text { (95\% CI) }\end{array}$ & p-value \\
\hline $1.01(1.00-1.03)$ & 0.100 & $1.01(1.00-1.02)$ & 0.163 & $1.00(0.97-1.03)$ & 0.962 & $1.00(0.97-1.02)$ & 0.719 \\
\hline $1.01(0.99-1.02)$ & 0.431 & $1.01(1.00-1.02)$ & $0.040^{*}$ & $1.01(0.99-1.04)$ & 0.328 & $1.02(1.00-1.03)$ & $0.039 *$ \\
\hline $1.28(0.33-4.94)$ & 0.724 & $1.09(0.61-1.94)$ & 0.771 & $1.08(0.23-5.01)$ & 0.920 & $1.09(0.39-3.06)$ & 0.870 \\
\hline $1.00(0.99-1.01)$ & 0.562 & $1.01(1.00-1.01)$ & 0.089 & $1.01(0.99-1.03)$ & 0.360 & $1.00(0.99-1.02)$ & 0.495 \\
\hline $1.00(0.99-1.01)$ & 0.917 & $1.00(1.00-1.01)$ & 0.317 & $1.00(0.99-1.02)$ & 0.590 & $1.00(0.99-1.02)$ & 0.968 \\
\hline $0.99(0.97-1.02)$ & 0.756 & $1.01(1.00-1.01)$ & 0.206 & $1.00(0.98-1.02)$ & 0.931 & $1.00(0.99-1.02)$ & 0.897 \\
\hline $0.99(0.96-1.01)$ & 0.357 & $1.02(1.01-1.03)$ & $<0.001^{*}$ & $1.03(1.01-1.06)$ & $0.004^{*}$ & $1.02(1.00-1.04)$ & $0.028 *$ \\
\hline $0.98(0.96-0.99)$ & $0.004^{*}$ & $0.98(0.97-0.99)$ & $<0.001 *$ & $0.96(0.94-0.98)$ & $0.001 *$ & $0.97(0.95-0.99)$ & $<0.001 *$ \\
\hline $1.01(0.99-1.03)$ & 0.381 & $1.00(0.98-1.01)$ & 0.500 & $1.01(0.97-1.04)$ & 0.713 & $1.01(0.98-1.03)$ & 0.679 \\
\hline $1.00(0.95-1.06)$ & 0.865 & $0.99(0.97-1.01)$ & 0.175 & $0.99(0.94-1.04)$ & 0.663 & $1.00(0.96-1.04)$ & 0.983 \\
\hline $1.00(0.97-1.03)$ & 0.992 & $0.97(0.96-0.98)$ & $<0.001 *$ & $0.95(0.92-0.98)$ & $<0.001 *$ & $0.95(0.93-0.97)$ & $<0.001 *$ \\
\hline $1.03(1.01-1.05)$ & $0.013^{*}$ & $1.02(1.01-1.04)$ & $0.002 *$ & $1.10(1.05-1.15)$ & $<0.001 *$ & $1.04(1.02-1.07)$ & $0.002^{*}$ \\
\hline $0.96(0.93-0.98)$ & $<0.001^{*}$ & $0.96(0.95-0.97)$ & $<0.001^{*}$ & $0.93(0.89-0.96)$ & $<0.001 *$ & $0.94(0.91-0.96)$ & $<0.001 *$ \\
\hline $1.41(1.06-1.87)$ & $0.020 *$ & $1.31(1.09-1.57)$ & $0.004^{*}$ & $1.72(1.09-2.71)$ & $0.020 *$ & $1.63(1.17-2.26)$ & $0.004^{*}$ \\
\hline
\end{tabular}

All groups referenced to infants that had no LRTI. Rrs: respiratory system resistance; Crs: respiratory system compliance; Re: expiratory resistance; $X_{\mathrm{e}}$ : expiratory reactance; $R$ i: inspiratory resistance; Rei: respiratory resistance at the end of inspiration; Ree: respiratory resistance at the end of expiration; $X_{\mathrm{e}}$ : expiratory reactance; $X_{\mathrm{i}}$ : inspiratory reactance; $X \mathrm{ei}$ : respiratory reactance at the end of inspiration; $X_{\text {ee: }}$ respiratory reactance at the end of expiration; $\Delta R$ : volume dependence of resistance; $\Delta X$ : volume dependence of reactance; $A X V$ : area within the reactance versus volume loops. Antenatal confounders included in the analysis: sex, length-for-age z-score, maternal smoking during pregnancy, ethnicity, maternal HIV status and gestational age. ${ }^{\#}: n=161 ;{ }^{\uparrow}: n=50 ;{ }^{+}: n=38 ;{ }^{\S}: n=87 . *: p<0.05$. 
TABLE 5 Odds ratios (OR) established for the optimal cut-off values the intra-breath impedance measures for the prediction of lower respiratory tract illness (LRTI) in the first year of life

\begin{tabular}{|c|c|c|c|c|c|c|c|c|c|}
\hline & \multirow{2}{*}{$\begin{array}{l}\text { Optimal } \\
\text { cut-off }\end{array}$} & \multicolumn{2}{|c|}{ Any LRTI ${ }^{\#}$} & \multicolumn{2}{|c|}{ Recurrent LRTI } & \multicolumn{2}{|c|}{ Hospitalised LRTI ${ }^{+}$} & \multicolumn{2}{|c|}{ Wheezy LRTI§ } \\
\hline & & OR $(95 \% \mathrm{CI})$ & p-value & OR $(95 \% \mathrm{CI})$ & p-value & OR $(95 \% \mathrm{CI})$ & p-value & OR $(95 \% \mathrm{CI})$ & $\mathrm{p}$-value \\
\hline$X e \mathrm{hPa} \cdot \mathrm{s} \cdot \mathrm{L}^{-1}$ & -13.53 & $1.99(1.38-2.88)$ & $<0.001 *$ & 2.05 (1.59-2.65) & $<0.00$ & $2.97(1.52-5.80)$ & 0.0 & $2.87(1.8$ & $<0.001 *$ \\
\hline$\Delta R \mathrm{hPa} \cdot \mathrm{s} \cdot \mathrm{L}^{-1}$ & 11.06 & $2.64(1.68-4.14)$ & $<0.001 *$ & $2.42(1.84-3.18)$ & $<0.001^{*}$ & $5.43(2.69-10.97)$ & $<0.001 *$ & $3.02(1.80-5.08)$ & $<0.001 *$ \\
\hline AXV hPa.s & 0.10 & $1.75(1.19-2.57)$ & $0.005^{*}$ & $1.51(1.14-1.99)$ & $0.004^{*}$ & $2.01(0.97-4.24)$ & 0.065 & $1.90(1.14-3.15)$ & $0.013^{*}$ \\
\hline
\end{tabular}

All groups referenced to infants that had no LRTI. Xe: expiratory reactance; $\Delta R$ : volume dependence of resistance; $\Delta X$ : volume dependence of reactance; $\mathrm{AXV}$ : area within the reactance versus volume loops. Lung function measures were selected based on the logistic regression (see table 4). ${ }^{*}: n=161 ;{ }^{\text {ๆ}}: n=50 ;{ }^{+}: n=38 ;{ }^{\S}: n=87 . *$ : $p<0.05$.

with COPD [14] compared with healthy controls, and this was attributed to the presence of mechanical inhomogeneity improving progressively during inspiration $[13,14]$. It is possible that the same mechanisms govern the changes in inspiratory $X$ in the symptom-free infants in the present study and the more negative $\Delta X$ during inspiration represents increased inhomogeneity of the peripheral airways. Consequently, the abnormal $\Delta \mathrm{X}$ and $\Delta R$ values together most likely reflect an inhomogeneous peripheral airway obstruction at functional residual capacity, which may be a risk factor in the development of LRTI in the following months; whether this is a result of structural alteration which increases respiratory disease susceptibility or another underlying subclinical disease process requires further investigation.

The association with early lung function and subsequent wheezing is consistent with that previously reported $[2,3,23]$. In smaller cohorts investigating association with early-life wheezing, maximal flow at functional residual capacity measured in sedated newborns was lower in those infants who subsequently had bronchiolitis and/or recurrent wheeze in the first 2 years of life $[3,23]$. Similarly, in the present study, infants with low lung function reflecting airflow limitation had 3.9 times greater odds of developing wheezing that is associated with LRTI. Additionally, a relationship between low lung function and severity and frequency of LRTI was established. $\Delta R$ was the strongest predictor of hospitalisation: infants who had $\Delta R$ above the cut-off had 5.4 times higher risk developing severe LRTI requiring hospitalisation. The conventional FOT measures (e.g. Rmean and Xmean at $16 \mathrm{~Hz}$ ) or the mechanical parameters $R$ rs and $C$ rs did not exhibit any predicting power.

The present study represents one of the largest infant cohorts and the first in a low- to middle-income setting to assess the interaction between lung function shortly after birth and development of LRTI in infancy. Although previous studies have reported the association of low lung function and respiratory disease risk in infants, they have been smaller with lower incidence of LRTI [2]. An inherent limitation of the current study may be that cut-off values for lung function measures are valid for the present cohort and other populations may require new reference values to be established in their own settings. Ideally, these should be tested on an independent cohort to see how widely applicable these cut-off values are, particularly as this cohort had a high burden of respiratory disease and a high exposure to risk factors associated with respiratory illness such as maternal smoking and maternal HIV infection. A further limitation is that not all LRTI episodes may have been ascertained. However, given the excellent cohort retention, careful and close follow-up and the strong, reliable surveillance systems established for LRTI [17], and the high incidence found, this does not appear to be of concern.

The present study demonstrates that the intra-breath measures of FOT ( $\Delta X, \Delta R, X \mathrm{e}$ and AXV) are able to detect changes in lung function that are associated with an increasing risk of LRTI in infants living in a high respiratory disease burden setting. The lung function changes suggest that subclinical airway flow limitation and mechanical inhomogeneity may contribute to this vulnerability. This study has hence identified the intra-breath impedance measures as useful outcomes for lung health in infants that can be obtained noninvasively, quickly and without sedation, making this technique a potentially useful tool in longitudinal studies. Follow-up of this cohort will provide insight into the benefit of intra-breath measures of FOT for longitudinal lung function assessment and the long-term impact of LRTI on lung function.

\section{Conclusions}

Impaired lung function as reflected by intra-breath impedance measurement shortly after birth increases risk of LRTI, wheezing and severe LRTI in infancy. This new technique provides a safe and useful assessment of lung function in unsedated infants that can be used longitudinally. 
Acknowledgements: We thank the study and clinical staff at Paarl Hospital, Mbekweni and Newman clinics (Drakenstein Child Health Study sites) as well as the CEO of Paarl Hospital, Breslau Kruger and the Western Cape Health Dept (Western Cape, South Africa) for their support of the study. We thank the families and children who participated in this study.

Author contributions: D. Czovek, D.M. Gray, Z. Hantos and H.J. Zar conceived the study; D.M. Gray, D. Czovek, G.L. Hall, P.D. Sly, Z. Hantos and H.J. Zar contributed to the study design and provided intellectual input; D.M. Gray, L. McMillan, J.A.M. Stadler and A. Vanker recruited patients and collected data; D.M. Gray and L. McMillan recorded measurement data; B.L. Radics, Z. Gingl, D. Czovek and Z. Hantos contributed to development of the novel infant FOT technique; D.M. Gray, D. Czovek, Z. Hantos and L. Turkovic processed the recordings and analysed the data; D.M. Gray and D. Czovek drafted the manuscript; all authors have seen and approved the submitted manuscript.

Conflict of interest: D.M. Gray has nothing to disclose. D. Czovek has nothing to disclose. L. McMillan has nothing to disclose. L. Turkovic has nothing to disclose. J.A.M. Stadler has nothing to disclose. A. Vanker reports grants from Bill and Melinda Gates Foundation (OPP1017641), Discovery Foundation, South African Thoracic Society AstraZeneca Respiratory Fellowship, National Research Fund, South Africa, CIDRI Clinical Fellowship, and MRC, South Africa, during the conduct of the study. B.L. Radics has nothing to disclose. Z. Gingl has nothing to disclose. G.L. Hall has nothing to disclose. P.D. Sly is a named inventor on a patent owned by the Telethon Kids Institute that covers this technology. The patent is licensed to Thorasys, Inc. (Canada); P.D. Sly receives no financial gain from this patent. H.J. Zar reports grants from Gates Foundation, National Research Foundation South Africa, SA Medical Research Council, Wellcome Trust and National Institutes of Health, H3 Africa, during the conduct of the study. Z. Hantos has a patent 2005903034 (A Method of Diagnosing a Respiratory Disease or Disorder or Monitoring Treatment of Same and a Devise for Use Therein) licensed to Thorasys P/L; Z. Hantos is named as an inventor on this patent owned by the Telethon Kids Institute, Perth, Australia, but receives no royalties or other payment from the patent or licensee.

Support statement: This study was supported by grants from the Wellcome Trust (098479/z/12/z; 204755/z/16/z), Bill and Melinda Gates Foundation (OPP1017641), Worldwide University Network Research Mobility Award, University of Cape Town equipment grant, the Hungarian Scientific Research Fund (105403). G.L. Hall is funded by the National Health and Medical Research Foundation of Australia (1025550). P.D. Sly is funded by the National Health and Medical Research Foundation of Australia (1002035). Funding information for this article has been deposited with the Crossref Funder Registry.

\section{References}

1 Turner SW, Palmer LJ, Rye PJ, et al. Infants with flow limitation at 4 weeks: outcome at 6 and 11 years. Am J Respir Crit Care Med 2002; 165: 1294-1298.

2 Martinez FD, Morgan WJ, Wright AL, et al. Diminished lung function as a predisposing factor for wheezing respiratory illness in infants. $N$ Engl J Med 1988; 319: 1112-1117.

3 Murray CS, Pipis SD, McArdle EC, et al. Lung function at one month of age as a risk factor for infant respiratory symptoms in a high risk population. Thorax 2002; 57 : 388-392.

4 Walker CL, Rudan I, Liu L, et al. Global burden of childhood pneumonia and diarrhoea. Lancet 2013; 381: $1405-1416$.

5 Zar HJ, Ferkol TW. The global burden of respiratory disease - impact on child health. Pediatr Pulmonol 2014; 49: 430-434.

6 World Health Organization. Global Surveillance, Prevention and Control of Chronic Respiratory Diseases: A Comprehensive Approach. 2007. www.who.int/respiratory/publications/global_surveillance/en/ Date last accessed: November 16, 2018

7 Chan JY, Stern DA, Guerra S, et al. Pneumonia in childhood and impaired lung function in adults: a longitudinal study. Pediatrics 2015; 135: 607-616.

8 Dezateux C, Stocks J, Wade AM, et al. Airway function at one year: association with premorbid airway function, wheezing, and maternal smoking. Thorax 2001; 56: 680-686.

9 Castro-Rodriguez JA, Holberg CJ, Wright AL, et al. Association of radiologically ascertained pneumonia before age $3 \mathrm{yr}$ with asthmalike symptoms and pulmonary function during childhood: a prospective study. Am J Respir Crit Care Med 1999; 159: 1891-1897.

10 Gray DM, Turkovic L, Willemse L, et al. Lung function in African infants in the Drakenstein Child Health Study. Impact of lower respiratory tract illness. Am J Respir Crit Care Med 2017; 195: 212-220.

11 Gray D, Willemse L, Visagie A, et al. Determinants of early-life lung function in African infants. Thorax 2016; 72: 445-450.

12 Gray D, Czovek D, Smith E, et al. Respiratory impedance in healthy unsedated South African infants: effects of maternal smoking. Respirology 2015; 20: 467-473.

13 Czovek D, Shackleton C, Hantos Z, et al. Tidal changes in respiratory resistance are sensitive indicators of airway obstruction in children. Thorax 2016; 71: 907-915.

14 Lorx A, Czovek D, Gingl Z, et al. Airway dynamics in COPD patients by within-breath impedance tracking: effects of continuous positive airway pressure. Eur Respir J 2017; 49: 1601270.

15 Zar HJ, Barnett W, Myer L, et al. Investigating the early-life determinants of illness in Africa: the Drakenstein Child Health Study. Thorax 2015; 70: 592-594.

16 Zar HJ, Barnett W, Stadler A, et al. Aetiology of childhood pneumonia in a well vaccinated South African birth cohort: a nested case-control study of the Drakenstein Child Health Study. Lancet Respir Med 2016; 4: 463-472.

17 le Roux DM, Myer L, Nicol MP, et al. Incidence of childhood pneumonia: facility-based surveillance estimate compared to measured incidence in a South African birth cohort study. BMJ Open 2015; 5: e009111.

18 World Health Organization. Integrated Management Of Childhood Illness: Distance Learning Course. Geneva, WHO, 2014.

19 Vanker A, Barnett W, Brittain K, et al. Antenatal and early life tobacco smoke exposure in an African birth cohort study. Int J Tuberc Lung Dis 2016; 20: 729-737. 
20 Stein DJ, Koen N, Donald KA, et al. Investigating the psychosocial determinants of child health in Africa: the Drakenstein Child Health Study. J Neurosci Methods 2015; 252: 27-35.

21 Hantos Z, Czovek D, Gyurkovits Z, et al. Assessment of respiratory mechanics with forced oscillations in healthy newborns. Pediatr Pulmonol 2015; 50: 344-352.

22 van der Ent CK, Brackel HJ, van der Laag J, et al. Tidal breathing analysis as a measure of airway obstruction in children three years of age and older. Am J Respir Crit Care Med 1996; 153: 1253-1258.

23 Young S, O'Keeffe PT, Arnott J, et al. Lung function, airway responsiveness, and respiratory symptoms before and after bronchiolitis. Arch Dis Child 1995; 72: 16-24. 